\title{
Geriatric Medicine and Gerontology
}

META ANALYSIS

\section{Meta-Analysis of Acute Sarcopenia among Hospitalized Elderly Patients}

\section{Arthur George Gonzales, MD* and Miguel Ramos, MD}

Geriatric Center St. Luke's Medical Center, Quezon City, Philippines

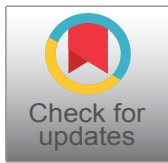

*Corresponding author: Arthur George Gonzales, Geriatric Center St. Luke's Medical Center, Quezon City, Philippines

\begin{abstract}
Objective: To determine the prevalence of acute sarcopenia in elderly hospitalised patients.

Search strategy: The electronic bibliographic databases used are MEDLINE via PUBMED and The Cochrane Library (Cochrane Database of Systematic Reviews, Cochrane Central Register of Controlled Trials (CENTRAL), Cochrane Methodology Register). The search strategy included a combination of appropriate MeSH and other free-text terms including the following key words: "sarcopenia", "acute care", "hospitalisation", and "elderly". There was no language restriction for the searches. Abstracts and subsequently selected full studies reporting the prevalence of sarcopenia in elderly adults admitted to inpatient hospitals were reviewed as long as the diagnosis of sarcopenia included at least the assessment of muscle mass.
\end{abstract}

Selection criteria: Observational studies involving elderly Patient $>65 \mathrm{y} / \mathrm{o}$, admitted in an acute care hospital with no sarcopenia. Diagnosis of sarcopenia upon discharged based on EWGSOP or AWGS definition.

Data collection and analysis: All published reports of all eligible studies were evaluated by two independent reviewers. Data were extracted data and pooled analysis was done on the different variables collected.

Main results: The present study identified six observational studies involving 2106 participants with 418 hospitalized elderly noted to have sarcopenia, showing a prevalence of $19.8 \%$. The age of elderly patients with sarcopenia was significantly higher than those without sarcopenia by 2.91 years $(95 \% \mathrm{Cl}, 2.18$ to 3.65$)$ while the $\mathrm{BMI}$ was significantly lower. No significant difference was noted in the proportion of male elderly with or without sarcopenia. A higher proportion of smokers were noted among those with sarcopenia. The risk of smokers for sarcopenia was $1.26 \mathrm{x}$ higher than non-smokers ( $\mathrm{RR}=1.26 ; 95 \% \mathrm{Cl}$ : 1.07-1.48). No significant difference was observed in the length of stay, but a higher risk for mortality $(\mathrm{RR}=2.69 ; 95 \% \mathrm{Cl}$ : $1.96-3.69)$ and readmission ( $\mathrm{RR}=1.48 ; 95 \% \mathrm{Cl}: 1.27-1.72$ ) was noted among hospitalized elderly patients with sarcopenia.
Conclusion: The results of this review showed that the prevalence of acute sarcopenia among elderly hospitalized patients was $19.8 \%$. The age of elderly patients with sarcopenia was significantly higher than those without sarcopenia while the BMI was lower. The proportion of smokers was also higher among elderly patients with sarcopenia. Moreover, the results of this meta-analysis showed that the length of stay.was not significantly different between elderly patients with or without sarcopenia. However, mortality and readmission rate were significantly higher among elderly patients with acute sarcopenia.

\section{Keywords}

Acute Sarcopenia, Elderly, Hospitalization

\section{Introduction}

Sarcopenia is a common disease associated with the ageing process wherein there is a degenerative loss of skeletal muscle mass, quality, strength and function that leads to physical disability, poor quality of life, functional decline, higher rate of fall, higher rate of hospitalizations, increase health care expenditures and death [1-4]. It was also found to be a consistent predictor of chronic disease progression, all-cause mortality, poorer functional outcomes, and higher postoperative complications [5].

The causes of sarcopenia in elderly population appear to be multifactorial and these are: Environmental causes, inflammation, disease triggers, hormonal changes, decrease nutrition and sedentary lifestyle [6]. Acute sarcopenia refers to acute loss of muscle mass and function associated with hospitalization and studies concerning the incidence of sarcopenia among older people admitted during hospital stay are few [7]. In older patients, besides the negative effect of the acute event, hospitalization itself might represent

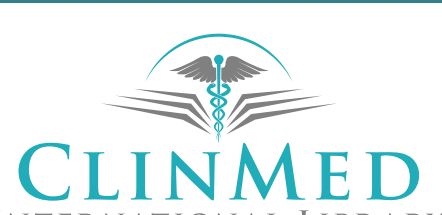

INTERNATIONAL LIBRARY
Citation: Gonzales AG, Ramos M (2021) Meta-Analysis of Acute Sarcopenia among Hospitalized Elderly Patients. J Geriatr Med Gerontol 7:126. doi.org/10.23937/2469-5858/1510126

Accepted: October 20, 2021: Published: October 22, 2021

Copyright: (c) 2021 Gonzales AG, et al. This is an open-access article distributed under the terms of the Creative Commons Attribution License, which permits unrestricted use, distribution, and reproduction in any medium, provided the original author and source are credited. 
an additional stressor. After 3 days of hospitalization, elderly inpatients lost approximately the same amount of lean leg muscle mass as healthy older subjects experienced in 10 days of inactivity-approximately three-fold greater loss of lean leg muscle mass than a younger cohort confined to bed for 28 days $[8,9]$.

Worldwide, the prevalence of sarcopenia is $10 \%$ and Asian people appear to have a higher prevalence of sarcopenia thanin any other regions. Its prevalence varies from $9.6-22.1 \%$ in men and $7.7-21.8 \%$ in women [10-12]. Majority of the studies regarding sarcopenia are in Europe and America. Current available studies in Asia have been published from eastern Asia, namely Japan, China, Taiwan, Korea and Thailand. The causes of sarcopenia in elderly population appears to be multifactorial, hence the objective of this study was to conduct a meta-analysis of reported prevalence of acute sarcopenia in elderly admitted patients.

\section{Objective}

\section{General objective}

The objective of this study was to conduct a metaanalysis of reported prevalence of acute sarcopenia in elderly hospitalised patients.

\section{Specific objectives}

1. To determine the prevalence of acute sarcopenia on elderly patients when admitted.

2. To identify risk factors for the development of sarcopenia at the time of admission and during hospitalization.

3. To determine the presence of acute sarcopenia among elderly patients with in-hospital outcomes i.e. length of hospital stay, length of bed rest, transfer to intensive units, hospital acquired pneumonia, mortality.

\section{Methods}

The meta-analysis was conducted according to the Preferred Reporting Items for Systematic Reviews and Meta-analysis (PRISMA) guidelines.

Type of study \& time period \& target population:

Type of study: Retrospective

- Target Population-Geriatric patients > 65-yearold admitted in an acute care hospital.

- Time Period - Studies published between January 1988 (chosen because the term "sarcopenia" was coined by Rosenberg in 1989) [13] and December 2019.

Inclusion criteria and Exclusion criteria for subject selection Inclusion criteria for the studies:

a) Elderly Patient $\geq 65 \mathrm{y} / \mathrm{o}$ b) Admitted in an acute care hospital with no sarcopenia

c) Diagnosis of sarcopenia upon discharged based on EWGSOP or AWGS definition

\section{Exclusion criteria:}

a) Bedridden patients upon admission

\section{Operational definitions:}

- Sarcopenia- as defined by the Asian Working Group for Sarcopenia (AWGS), muscle mass measurements of $7.0 \mathrm{~kg} / \mathrm{m} 2$ for men and $5.7 \mathrm{~kg} /$ $\mathrm{m} 2$ for women by using bioimpedance analysis, handgrip strength ( $<26 \mathrm{~kg}$ for men and $<18 \mathrm{~kg}$ for women), and usual gait speed $(<0.8 \mathrm{~m} / \mathrm{s})$.

\section{Description of study procedure}

The electronic bibliographic databases used are MEDLINE via PUBMED and The Cochrane Library (Cochrane Database of Systematic Reviews, Cochrane Central Register of Controlled Trials (CENTRAL), Cochrane Methodology Register). The search strategy included a combination of appropriate MeSH and other free-text terms including the following key words: "sarcopenia", "acute care", "hospitalisation", and "elderly". There was no language restriction for the searches provided the abstracts are available in English. Abstracts and subsequently selected full studies reporting the prevalence of sarcopenia in elderly adults admitted to inpatient hospitals were reviewed irrespective of design, as long as the diagnosis of sarcopenia included at least the assessment of muscle mass.

Data were extracted independently by the reviewer according to a standardized data extraction form. The following data were extracted: Study population, participant demographics and baseline characteristics, reported prevalence of sarcopenia, method of sarcopenia diagnosis, and study methodology.

\section{Data analysis}

Overall effect for each meta-analysis was derived by using both random effects model. Whenever needed, a fixed effects model was utilized. For the outcomes mortality and readmission, since this is a binary measure, the pooled statistics were reported as the odds ratio (OR) between the experimental and control groups with 95\% confidence intervals (CI). Other outcomes stated in the objective are transfer to intensive units and hospital acquired pneumonia, however, these were not reported in all the studies included in this meta-analysis. For binary outcomes, the number of subjects with an event and the total number of subjects in the group were extracted from the individual study. For numerical data, length of stay and length of bed rest, the mean and SD was used to calculate the mean difference (MD). Pooled estimates of the weighted mean differences and $95 \% \mathrm{Cl}$ were calculated using either a random effects 


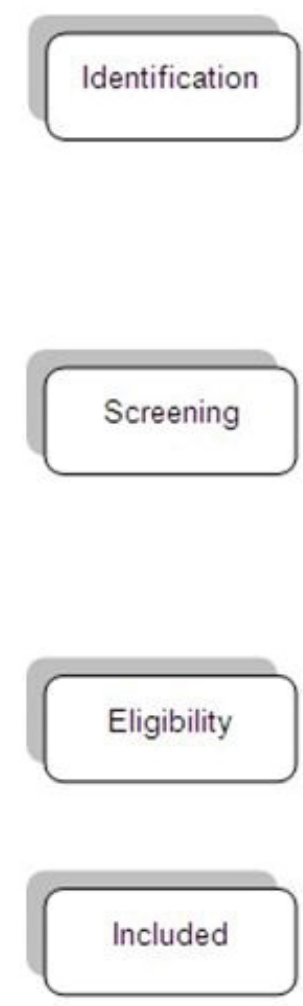

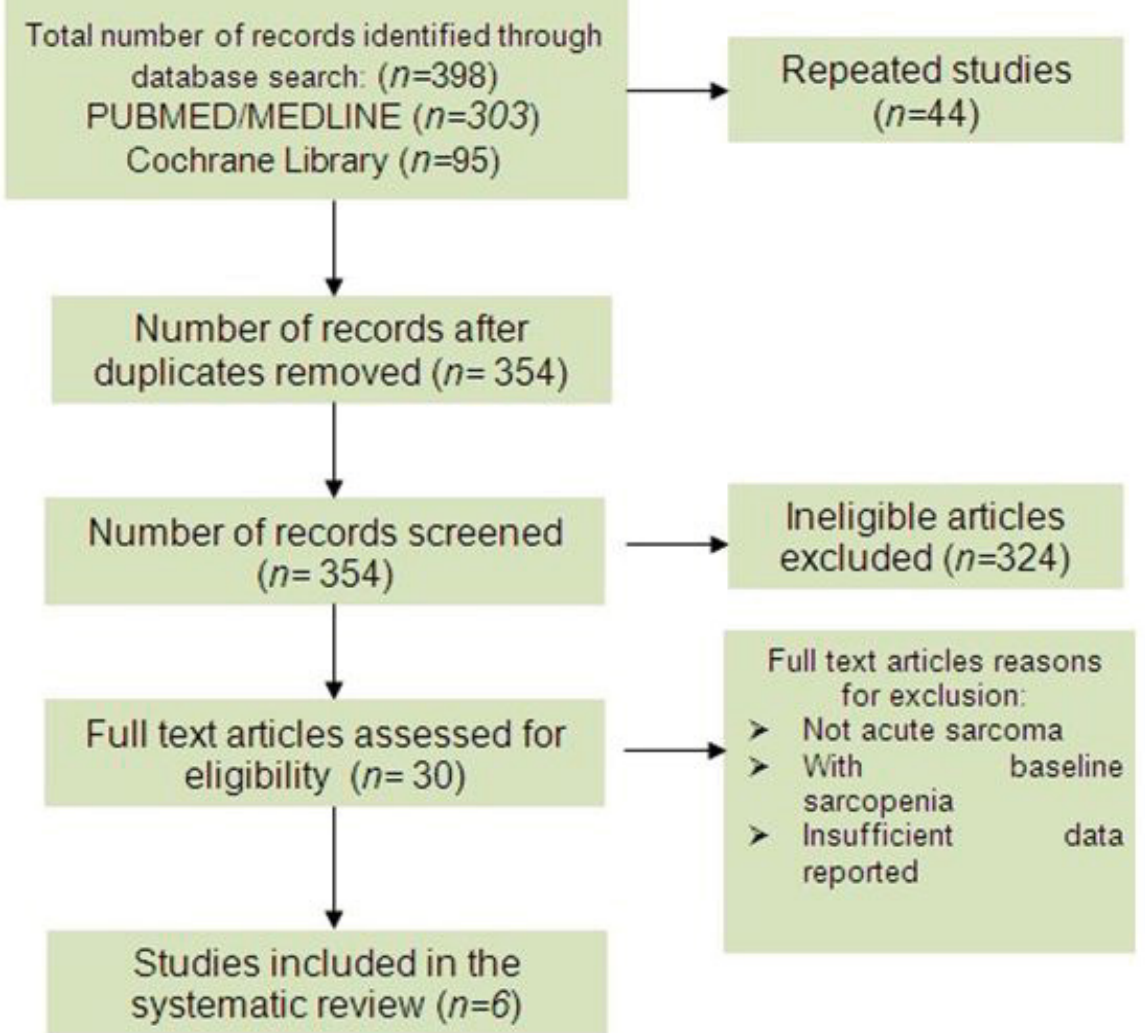

Figure 1: Flow chart for article selection. model if heterogeneity was found among studies, otherwise, a fixed-effects model was utilized. Statistical heterogeneity between studies was evaluated using Cochran's $Q$ test and I-squared [14]. P values $\leq 0.05$ was considered statistically significant. All meta-analyses were performed both with a fixed-effect and a randomeffects model. Review Manager 5.2 (RevMan 5.2) was utilized in the analysis.

\section{Results}

The literature search and review retrieved a total of 398 citations (Figure 1). After screening of publications, 354 remaining papers were screened for eligibility. Of these, 324 were further excluded. The text of the remaining 30 citations was analyzed further in detail and only 6 met the inclusion criteria and were included in the meta-analysis [4,14-18].

\section{Study characteristics}

The present study identified six studies determining the prevalence of acute sarcopenia among hospitalized elderly. Table 1, Table 2 and Table 3 summarizes the characteristics of the included observational studies. Six studies [4,14-18] involving 2106 participants (418 with sarcopenia and 1665 without sarcopenia) met the inclusion criteria.

All six studies were observational studies. One study had subjects from a previous RCT, 1 is multicenter observational study and the others were all prospective observational studies. All participants were elderly. Although one study included elderly aged $\geq 60$ years, however, the minimum age was $>65$-years-old.

A total of 418 participants were noted to have sarcopenia, showing a prevalence of $19.8 \%$. In one study [15], 23 had uncertain diagnosis. The diagnosis of sarcopenia was based on the algorithm proposed by the European Working Group on Sarcopenia in Older People (EWGSOP) or the AWGS. Muscle mass was quantified with the bioelectrical impedance analysis (BIA) and estimated using the equation developed by Janssen \& Colleagues in four studies, while one study assessed muscle mass by measuring mid-arm muscle circumference (MAMC). Another study assessed muscle mass by measuring Appendicular Skeletal Muscle Mass (ASM) using validated equation in Chinese population. Muscle strength was assessed using handgrip dynamometer while physical performance was assessed using gait speed $(4 \mathrm{~m})$.

Outcomes analyzed included length of stay, length of bed rest, transfer to intensive units, hospital acquired pneumonia, and mortality. However, none of the studies reported transfer to intensive units and hospital acquired pneumonia. Readmission was also collected and included in the outcome of the study.

\section{Quality assessment}

The methodology of each included trial was evaluated using the New Castle-Ottawa Scale (NOS). Of 


\begin{tabular}{|c|c|c|c|c|c|c|}
\hline 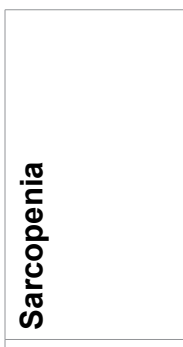 & 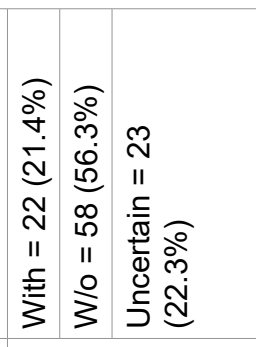 & 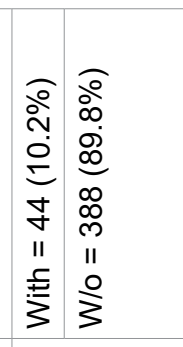 & 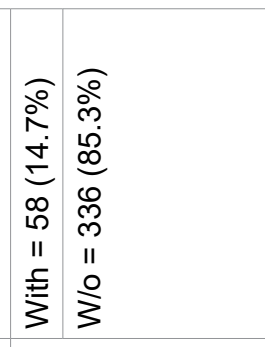 & 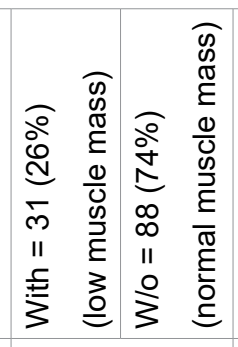 & 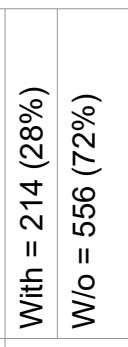 & 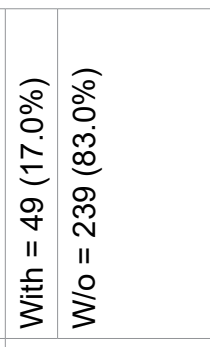 \\
\hline 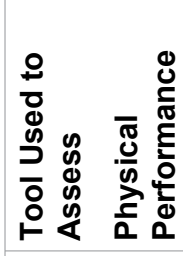 & 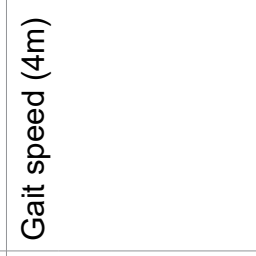 & 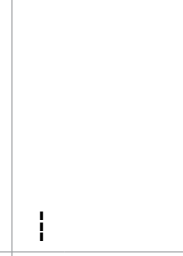 & 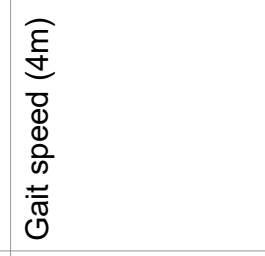 & 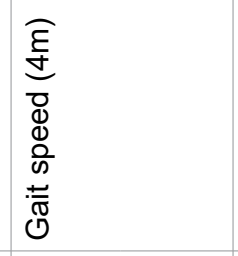 & 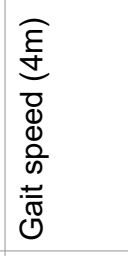 & 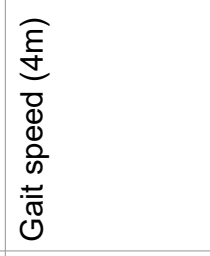 \\
\hline 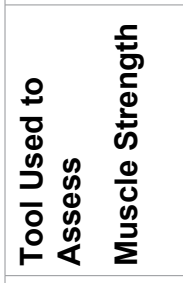 & 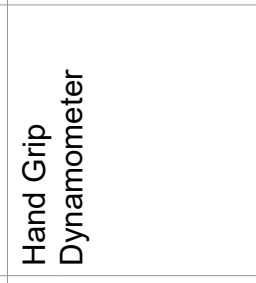 & 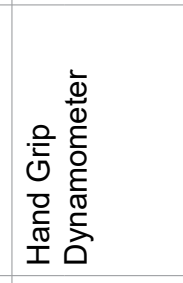 &  & 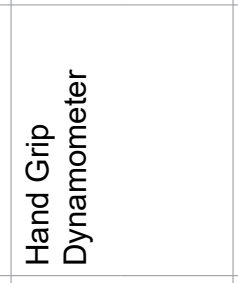 & 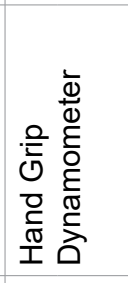 & 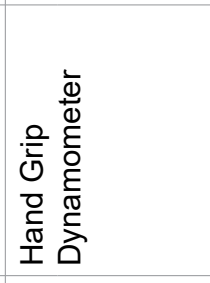 \\
\hline 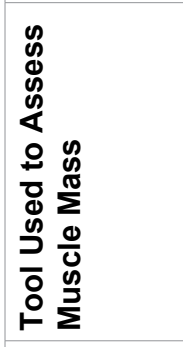 & 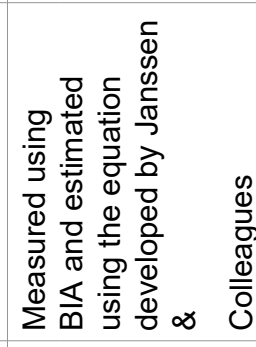 & 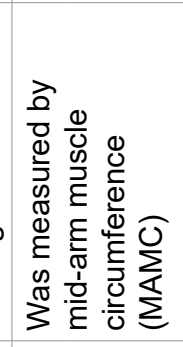 & 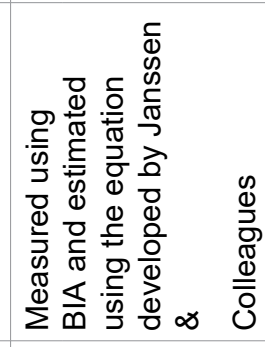 & 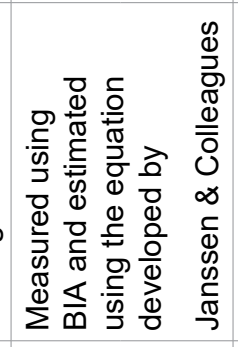 & 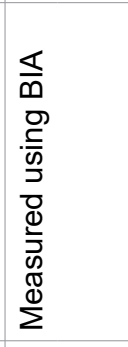 & 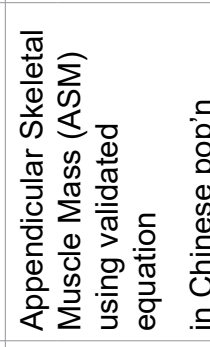 \\
\hline 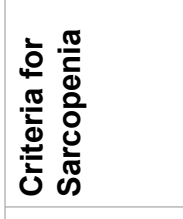 & $\begin{array}{l}0 \\
0 \\
0 \\
0 \\
\sum_{4}\end{array}$ & $\begin{array}{l}0 \\
0 \\
0 \\
0 \\
\text { J } \\
\text { W }\end{array}$ & $\begin{array}{l}0 \\
0 \\
0 \\
0 \\
\text { ju }\end{array}$ & 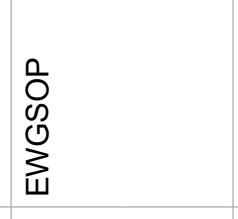 & $\begin{array}{l}0 \\
0 \\
0 \\
0 \\
3 \\
w\end{array}$ & $\begin{array}{l}\infty \\
0 \\
\sum_{<}\end{array}$ \\
\hline$=$ & $\stackrel{\Re}{\circ}$ & $\stackrel{\widetilde{F}}{\mathscr{\gamma}}$ & 胥 & $\stackrel{g}{\rightleftharpoons}$ & $\stackrel{R}{R}$ & $\stackrel{\infty}{\infty}$ \\
\hline$\stackrel{2}{\gtrless}$ & 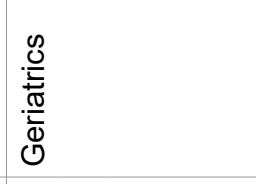 & 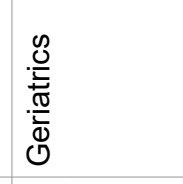 & 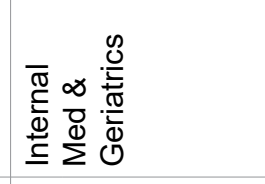 & 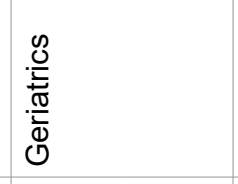 & 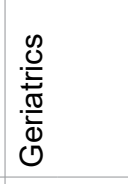 & 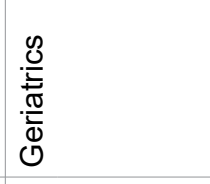 \\
\hline 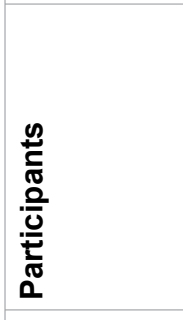 & 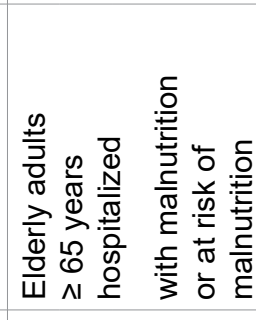 & 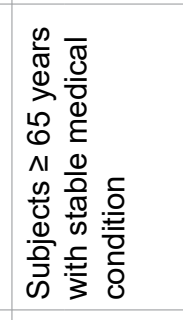 & 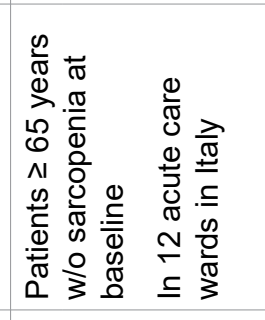 & 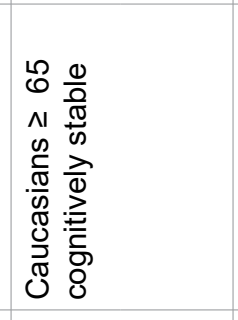 & 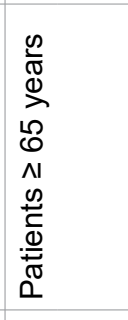 & 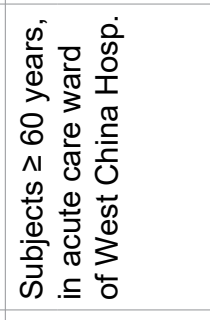 \\
\hline 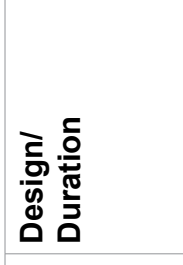 & 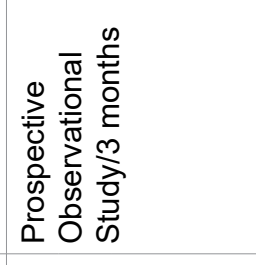 &  & 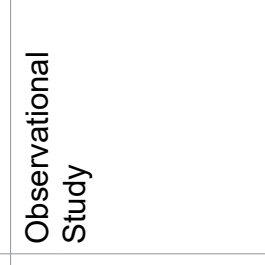 & 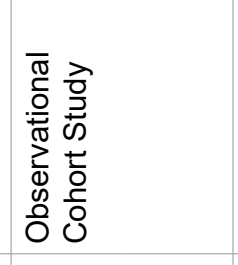 & 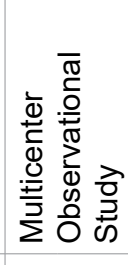 & 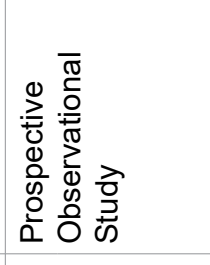 \\
\hline 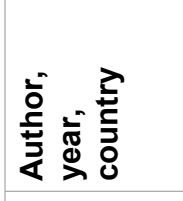 & 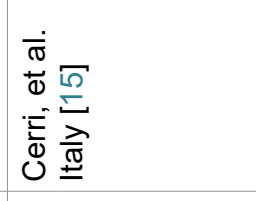 & 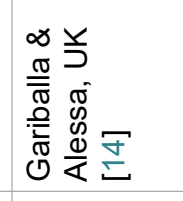 & 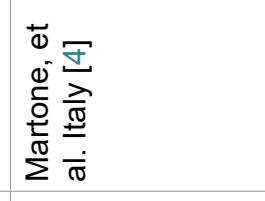 & 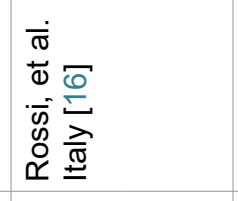 &  & 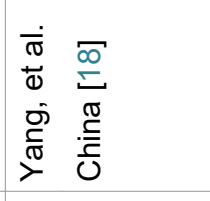 \\
\hline 0 & - & $N$ & $m$ & ナ & ما & 0 \\
\hline
\end{tabular}




\section{高旁}

เ

in $\stackrel{\text { ก }}{2}$

๕⿺

$F R$

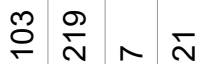

\&

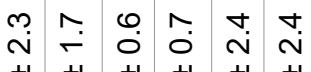

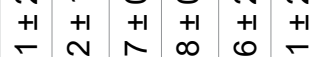

亏

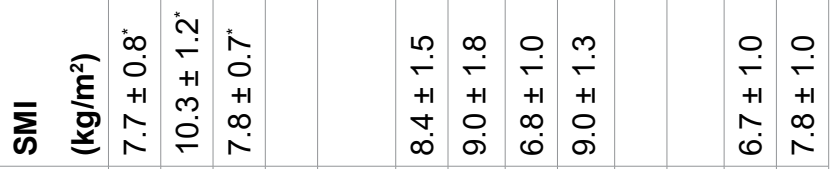

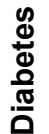

$\sim \stackrel{2}{2}$

$\cong \stackrel{+}{\frac{2}{2}}$

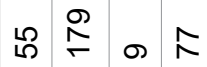

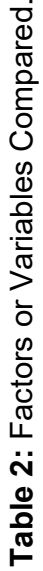

\begin{tabular}{|c|c|c|c|c|c|c|}
\hline$\frac{z}{\text { za }}$ & & & & & 8 & \\
\hline 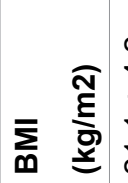 & & & & & & \\
\hline
\end{tabular}

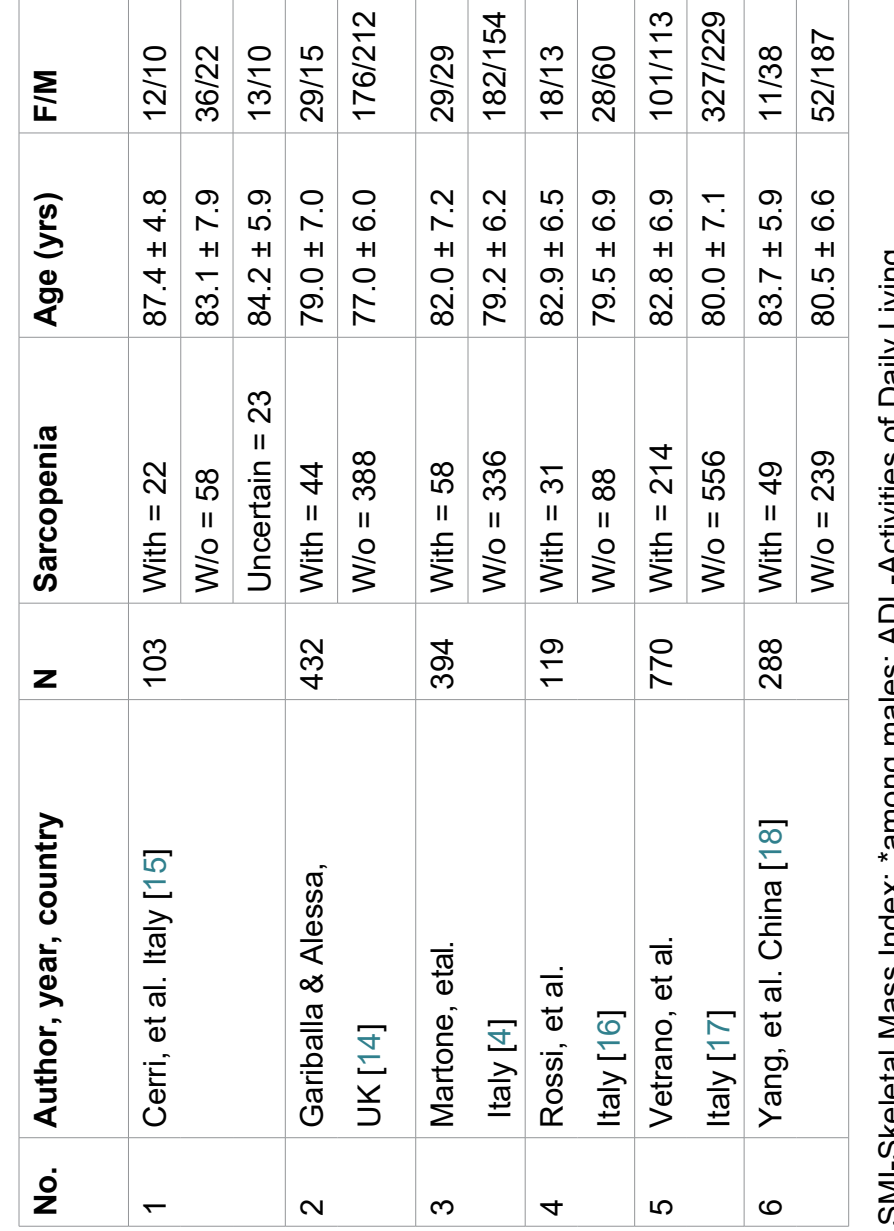

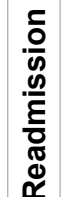

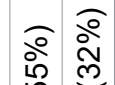

○๐ 它 $1,1, \frac{a}{0}$ 字

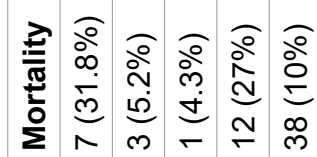

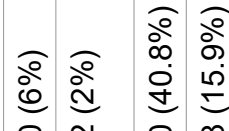

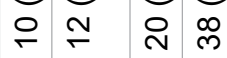



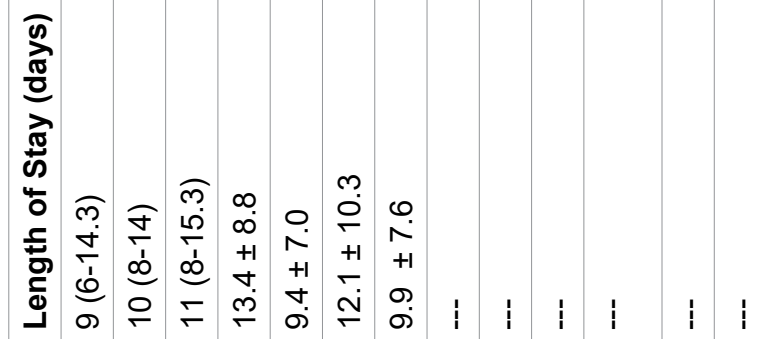

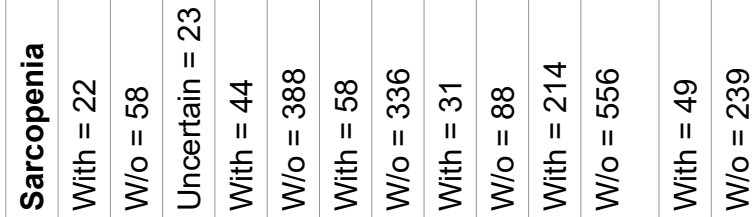

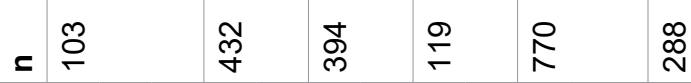

\begin{tabular}{|c|c|c|c|c|}
\hline 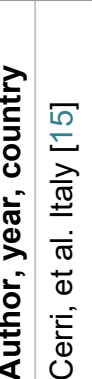 & 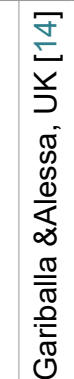 & 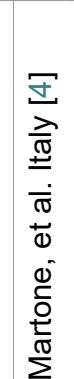 & 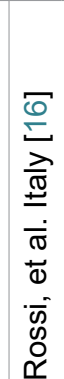 & 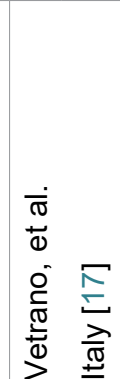 \\
\hline
\end{tabular}


Table 4: NOS Scores.

\begin{tabular}{|l|l|l|l|l|l|}
\hline No. & Author, year, country & $\begin{array}{l}\text { Selection } \\
\mathbf{( 4 ~ s t a r s )}\end{array}$ & $\begin{array}{l}\text { Comparability } \\
\mathbf{( 2 ~ s t a r s )}\end{array}$ & $\begin{array}{l}\text { Outcome/Exposure } \\
\text { (3 stars) }\end{array}$ & $\begin{array}{l}\text { Total Score } \\
\text { (9 stars) }\end{array}$ \\
\hline $\mathbf{1}$ & Cerri, et al. Italy [15] & 3 & $\mathbf{2}$ & 2 & 7 \\
\hline $\mathbf{2}$ & Gariballa \& Alessa, UK [14] & 3 & 2 & 3 & 8 \\
\hline 3 & Martone, et al. Italy [4] & 4 & 2 & 2 & 8 \\
\hline 4 & Rossi, et al. Italy [16] & 3 & 2 & 2 & 7 \\
\hline 5 & Vetrano, et al, Italy [17] & 3 & 1 & 3 & 7 \\
\hline 6 & Yang, et al. China [18] & 3 & 2 & 3 & 8 \\
\hline
\end{tabular}

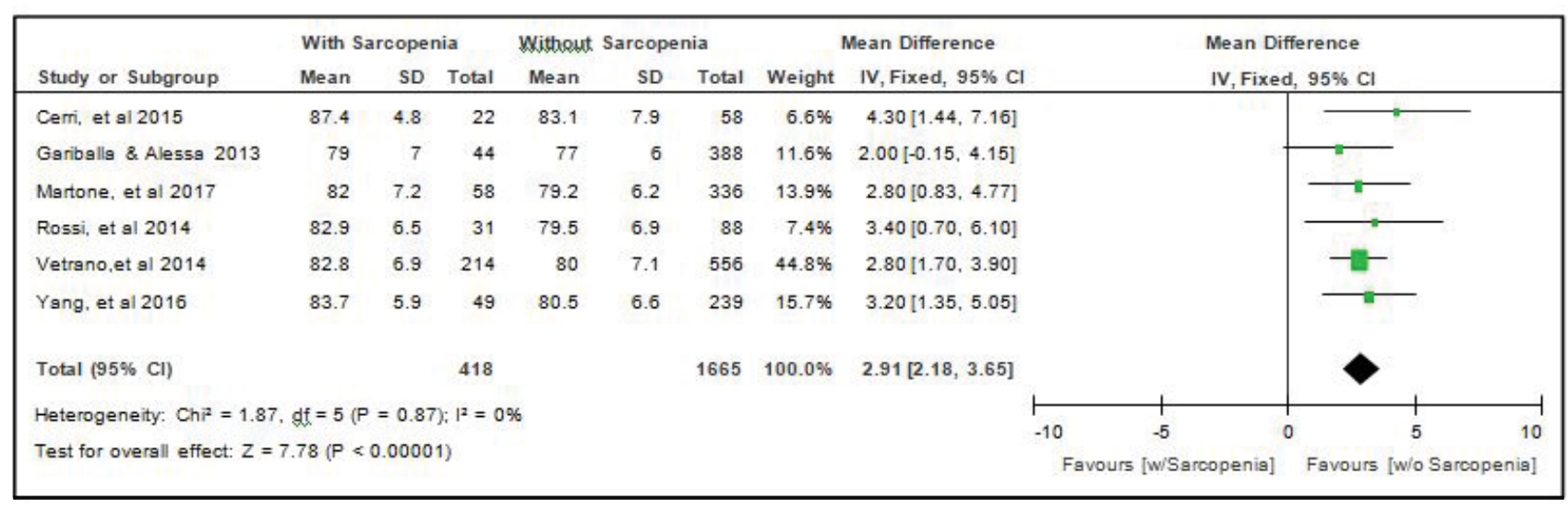

Figure 2: Comparison of age between patients with or without sarcopenia (fixed effects model).

\begin{tabular}{|c|c|c|c|c|c|c|c|}
\hline \multirow[b]{2}{*}{ Study or Subgroup } & \multicolumn{2}{|c|}{ With Sarcopenia } & \multicolumn{2}{|c|}{ Without Sarcopenia } & \multirow[b]{2}{*}{ Weight } & \multirow{2}{*}{$\begin{array}{c}\text { Risk Ratio } \\
\text { M-H, Random, } 95 \% \mathrm{Cl}\end{array}$} & \multirow{2}{*}{$\begin{array}{c}\text { Risk Ratio } \\
\text { M-H, Random, } 95 \% \text { C }\end{array}$} \\
\hline & Events & Total & Events & Total & & & \\
\hline Cerri, et al 2015 & 10 & 22 & 22 & 58 & $9.6 \%$ & $1.20[0.68,2.11]$ & -1 \\
\hline Grribslls \& Alessg 2013 & 15 & 44 & 212 & 388 & $13.4 \%$ & $0.62[0.41,0.95]$ & \\
\hline Martone, et sl 2017 & 29 & 58 & 154 & 336 & $18.3 \%$ & $1.09[0.82,1.45]$ & \\
\hline Rossi, et al 2014 & 13 & 31 & 60 & 88 & $12.9 \%$ & $0.62[0$ & \\
\hline Vetrano,et sl 2014 & 113 & 214 & 229 & 556 & $23.0 \%$ & & \\
\hline Yang, et al 2016 & 38 & 49 & 187 & 239 & & & \\
\hline Total $(95 \% \mathrm{Cl})$ & & 418 & & & & & \\
\hline Total events & 218 & & & & & & \\
\hline Heterogeneity: $T_{s u^{2}}=0.0$ & & & & & & & \\
\hline Test for overs & & & & & & & \\
\hline
\end{tabular}

Figure 3: Comparison of the distribution of male elderly patients with or without sarcopenia (random effects model).

the 6 citations included, three studies scored 7/9 [1517] on the NOS, and 3 scored $8 / 9[4,14,18]$, indicating good quality (Table 4).

\section{Synthesis of results (pooled analysis)}

Comparison of age of elderly patients with or without sarcopenia: All six studies [4,14-18] compared age of elderly hospitalized patients with or without sarcopenia and the analysis of pooled data demonstrated that there was a significant difference noted using the fixed effects model ( $Z=7.78 ; p<000001)$ (Figure 2$)$. The age of elderly patients with sarcopenia was significantly higher than those without sarcopenia by 2.91 years $(95 \% \mathrm{Cl}$, 2.18 to 3.65). A fixed effects model was used because there was no significant $(p=0.87)$ heterogeneity noted in the studies included and the $\mathrm{I}_{2}=0 \%$.
Comparison of the proportion of male elderly patients with or without sarcopenia: Again all six studies [4,14-18] compared data on the sex distribution of elderly hospitalized patients with or without sarcopenia and the analysis of pooled data demonstrated that there was no significant difference noted in the proportion of males elderly with or without sarcopenia using the random effects model $(Z=0.33 ; p=0.74)$ (Figure $3)$. There was a significant ( $p=0.003$ ) heterogeneity noted in the studies included and the $I_{2}=73 \%$, hence, a random effects model was preferred in the analysis over the fixed effects model.

Comparison of BMI of elderly patients with or without sarcopenia: Four studies $[4,15,16,18]$ compared BMI of elderly hospitalized patients with 


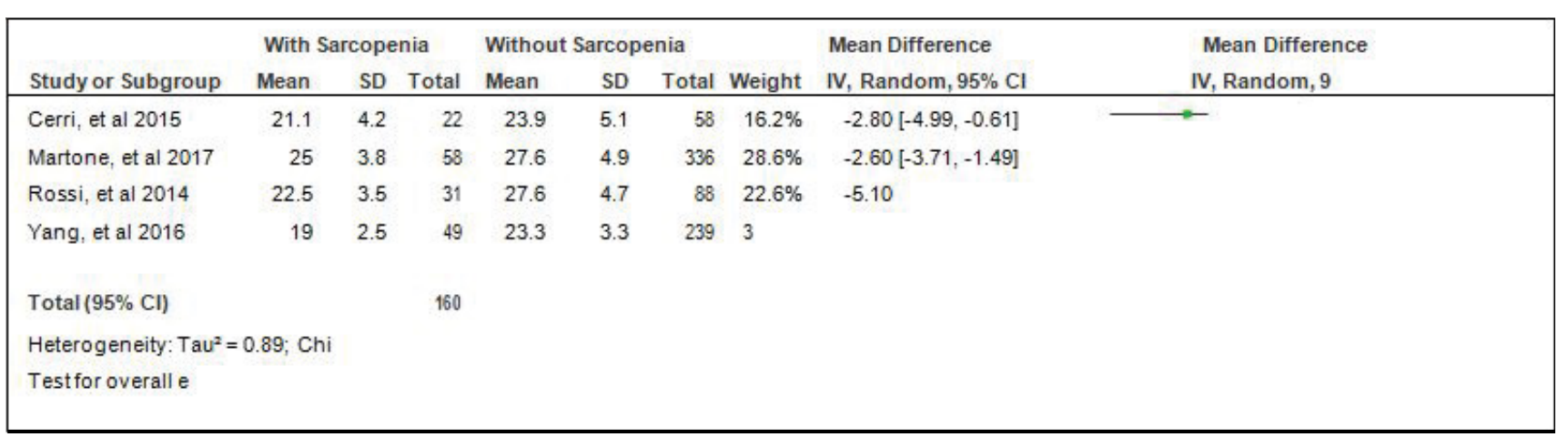

Figure 4: Comparison of BMI between patients with or without sarcopenia (random effects model).

\begin{tabular}{|c|c|c|c|c|c|c|c|}
\hline \multirow[b]{2}{*}{ Study or Subgroup } & \multicolumn{2}{|c|}{ With Sarcopenia } & \multicolumn{2}{|c|}{ Without Sarcopenia } & \multirow[b]{2}{*}{ Weight } & \multirow{2}{*}{$\begin{array}{c}\text { Risk Ratio } \\
\text { M-H, Fixed, } 95 \% \mathrm{Cl}\end{array}$} & \multirow{2}{*}{$\begin{array}{r}\text { Risk Ratio } \\
\text { M-H, Fixed, } 9 \\
\end{array}$} \\
\hline & Events & Total & Events & Total & & & \\
\hline Martone, et al 2017 & 38 & 58 & 256 & 336 & $20.6 \%$ & $0.86[0.71,1.05]$ & \\
\hline Vetrano,et al 2014 & 154 & 214 & 429 & 556 & $65.3 \%$ & $0.93[0.85,1.03]$ & \\
\hline Ysng, et al 2016 & 23 & 49 & 151 & 239 & $14.1 \%$ & 0.7 & \\
\hline Total $(95 \% \mathrm{Cl})$ & & 321 & & & & & \\
\hline Total events & 215 & & & & & & \\
\hline \multicolumn{8}{|c|}{ Heterogeneity: $C h i^{2}=2.31, \mathrm{df}$} \\
\hline Test for overall e & & & & & & & \\
\hline
\end{tabular}

Figure 5: Comparison of HPN between patients with or without sarcopenia (fixed effects model).

\begin{tabular}{|c|c|c|c|c|c|c|c|}
\hline \multirow[b]{2}{*}{ Study or Subgroup } & \multicolumn{2}{|c|}{ With Sarcopenia } & \multicolumn{3}{|c|}{ Without Sarcopenia } & \multirow{2}{*}{$\begin{array}{c}\text { Risk Ratio } \\
\text { M-H, Fixed, } 95 \% \mathrm{Cl}\end{array}$} & \multirow{2}{*}{$\begin{array}{c}\text { Risk Ratio } \\
\text { M-H, Fixed, 95\% }\end{array}$} \\
\hline & Events & Total & Events & Total & Weight & & \\
\hline Cerri, et al 2015 & 2 & 22 & 15 & 58 & $4.9 \%$ & $0.35[0.09,1.41]$ & - \\
\hline Martone, et al 2017 & 12 & 58 & 114 & 336 & $620.0 \%$ & $0.61[0.36,1.03]$ & \\
\hline Vetrano,et al 2014 & 55 & 214 & 179 & 556 & $659.4 \%$ & $0.80[0.62$ & \\
\hline Yang, et al 2016 & 9 & 49 & 77 & 239 & $915.6 \%$ & & \\
\hline Total $(95 \% \mathrm{Cl})$ & & 343 & & & & & \\
\hline Total events & 78 & & & & & & \\
\hline \multicolumn{8}{|c|}{ Heterogeneity: $\mathrm{Chi}^{2}=2.61, \mathrm{~d}$} \\
\hline Test for overall & & & & & & & \\
\hline
\end{tabular}

Figure 6: Comparison of diabetes between patients with or without sarcopenia (fixed effects model).

or without sarcopenia and the analysis of pooled data demonstrated that there was a significant difference noted using the fixed effects model $(Z=7.78$; $p<$ 000001) (Figure 4). The BMI of elderly patients with sarcopenia was significantly lower than those without sarcopenia by $3.75(95 \% \mathrm{Cl},-4.90$ to -2.60$)$. A random effects model was used because there was a significant ( $p=0.03$ ) heterogeneity noted in the studies included and the $\mathrm{I}_{2}=68 \%$.

Comparison of HPN between elderly patients with or without sarcopenia: Three studies $[4,17,18]$ compared data on the proportion hypertension among elderly hospitalized patients with or without sarcopenia, and the analysis of pooled data demonstrated that there was a significant difference noted in the proportion of hypertension among elderly with or without sarcopenia
$(Z=2.66 ; p=0.008)$ (Figure 5). The study showed that a lower proportion of patients with hypertension was noted among those with sarcopenia than those without sarcopenia which does not indicate that its a risk factor as shown by the RR that is $<1$ (RR=0.89; $95 \% \mathrm{Cl}$ : $0.82-$ 0.97). A fixed effects model was used because there was no significant $(p=0.32)$ heterogeneity noted in the studies included although the $\mathrm{I}_{2}=13 \%$.

Comparison of diabetes between elderly patients with or without sarcopenia: Four studies $[4,15,17,18]$ compared data on the proportion diabetes among elderly hospitalized patients with or without sarcopenia, and the analysis of pooled data demonstrated that there was a significant difference noted in the proportion of hypertension among elderly with or without sarcopenia $(Z=3.22 ; p=0.001)$ (Figure 6$)$. The study showed that 
a lower proportion of patients with diabetes was noted among those with sarcopenia than those without sarcopenia ( $R R=0.70 ; 95 \% \mathrm{Cl}: 0.57-0.87)$. A fixed effects model was used because there was no significant $(p=$ $0.46)$ heterogeneity noted in the studies included and the $\mathrm{I}_{2}=0 \%$.

Comparison of Skeletal Mass Index (SMI) of elderly patients with or without sarcopenia: Four studies $[4,15,17,18]$ compared SMI of elderly hospitalized patients with or without sarcopenia and the analysis of pooled data demonstrated that there was a significant difference noted using the random effects model $(Z=$ 3.67; $p=0.0002$ ) (Figure 7). The SMI of elderly patients with sarcopenia was significantly lower than those without sarcopenia by $1.62(95 \% \mathrm{Cl},-2.48$ to -0.75$)$. A random effects model was used because there was a significant $(p<0.00001)$ heterogeneity noted in the studies included and the $\mathrm{I}_{2}=95 \%$.

Comparison of Activities Of Daily Living (adl) of elderly patients with or without sarcopenia: Three studies $[4,16,17]$ compared ALD of elderly hospitalized patients with or without sarcopenia and the analysis of pooled data demonstrated that there was no significant difference noted using the random effects model $(Z$ $=1.32 ; \mathrm{p}=0.19$ ) (Figure 8). $\mathrm{A}$ random effects model was used because there was a significant $(p-0.002)$ heterogeneity noted in the studies included and the $I_{2}$ $=84 \%$.

Comparison of smoking between elderly patients with or without sarcopenia: Three studies $[14,17,18]$ compared data on the proportion smokers among elderly hospitalized patients with or without sarcopenia, and the analysis of pooled data demonstrated that there was a significant difference noted in the proportion of

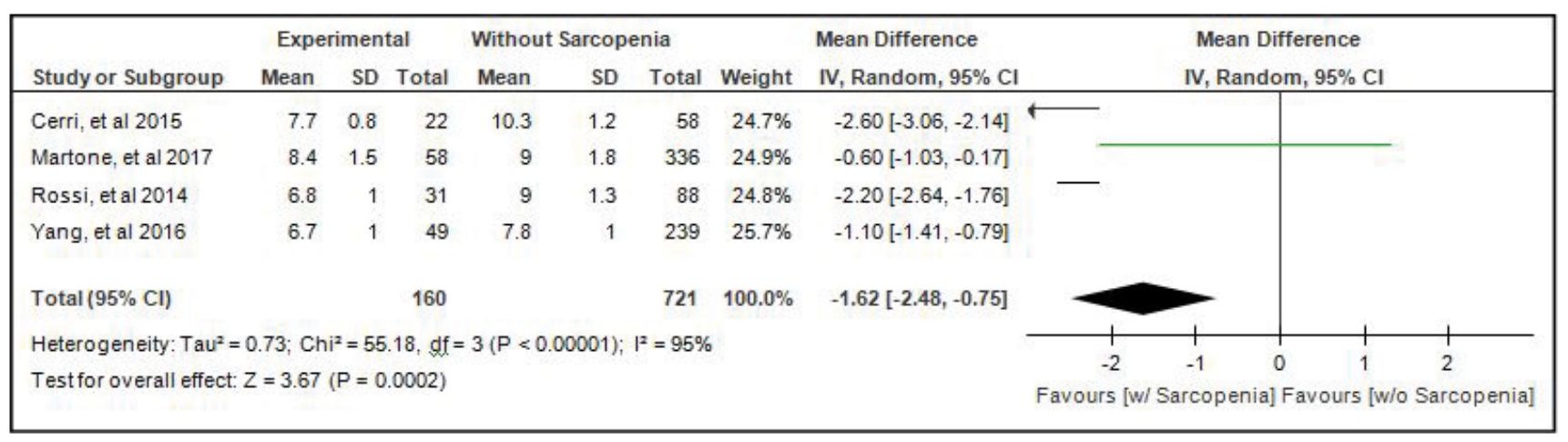

Figure 7: Comparison of skeletal mass index (smi) between patients with or without sarcopenia (random effects model).

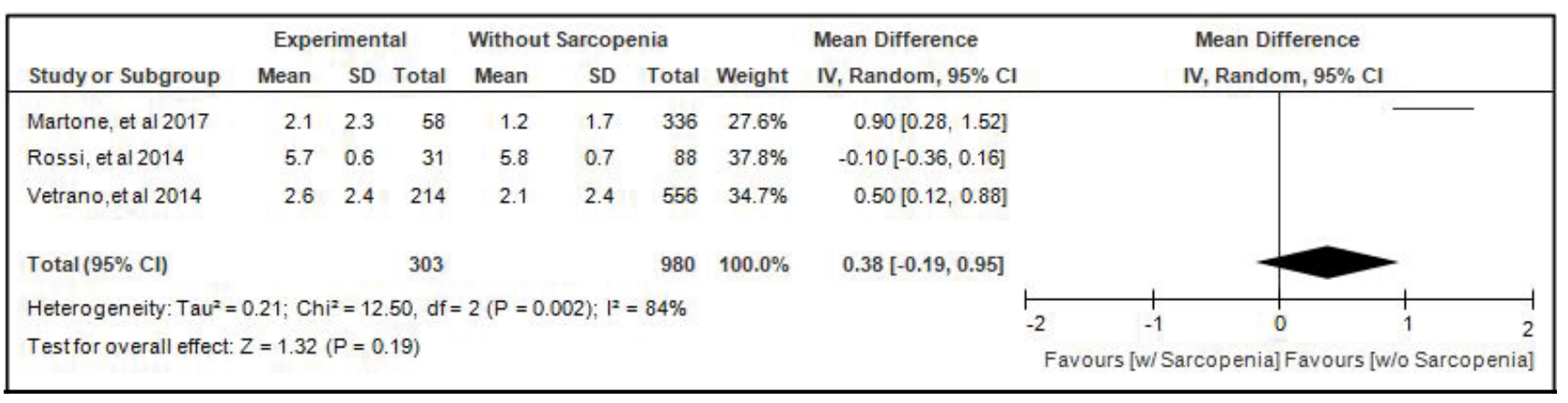

Figure 8: Comparison of activities of daily living (adl) between patients with or without sarcopenia (random effects model).

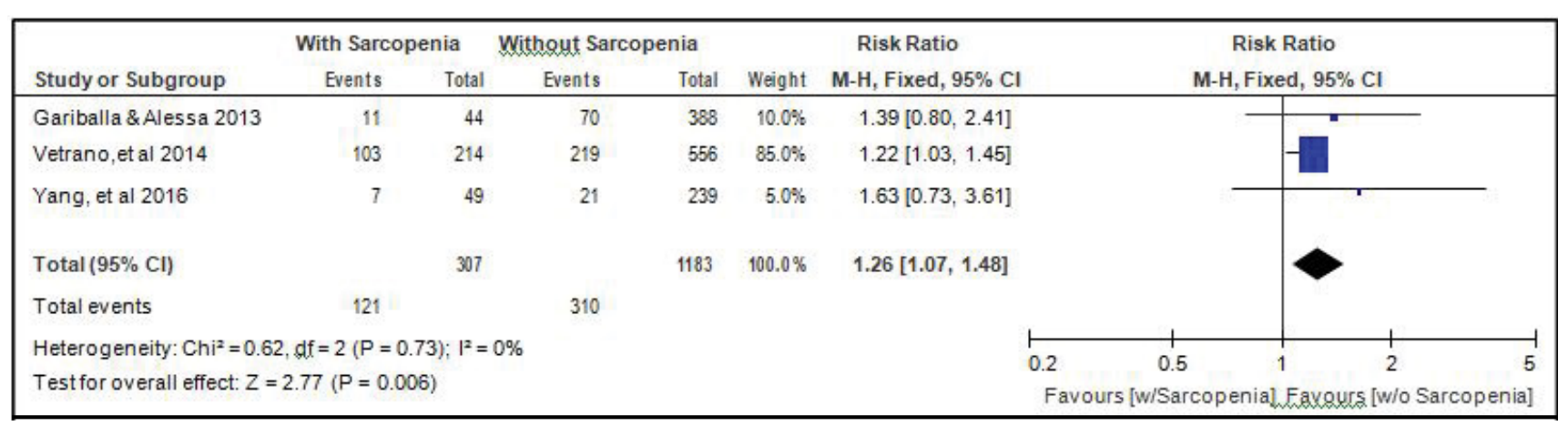

Figure 9: Comparison of smoking between patients with or without sarcopenia (fixed effects model). 


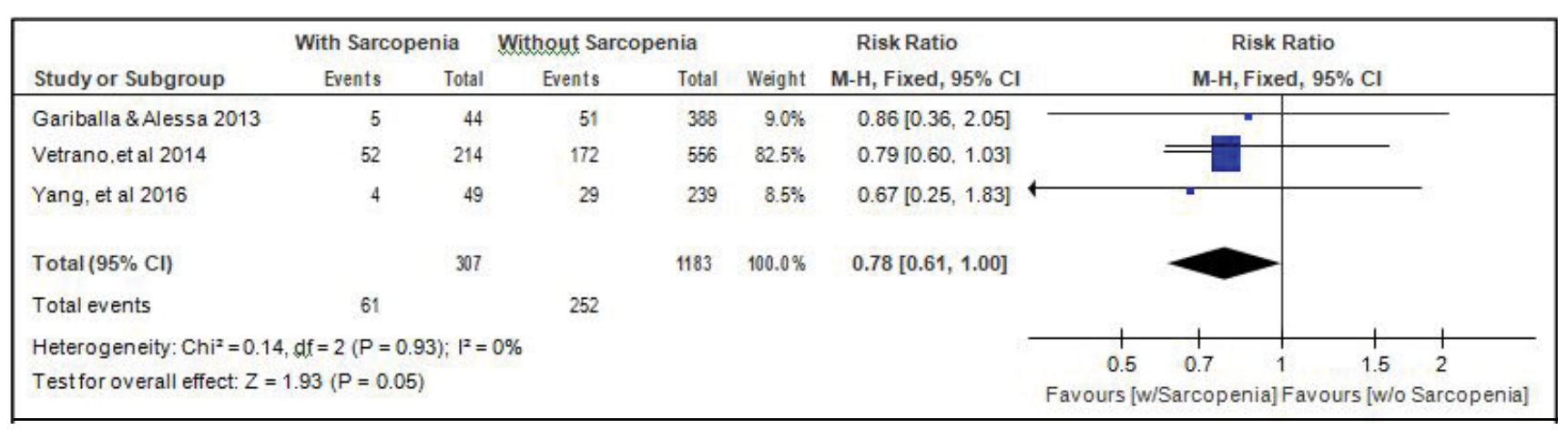

Figure 10: Comparison of alcohol drinking between patients with or without sarcopenia (fixed effects model).

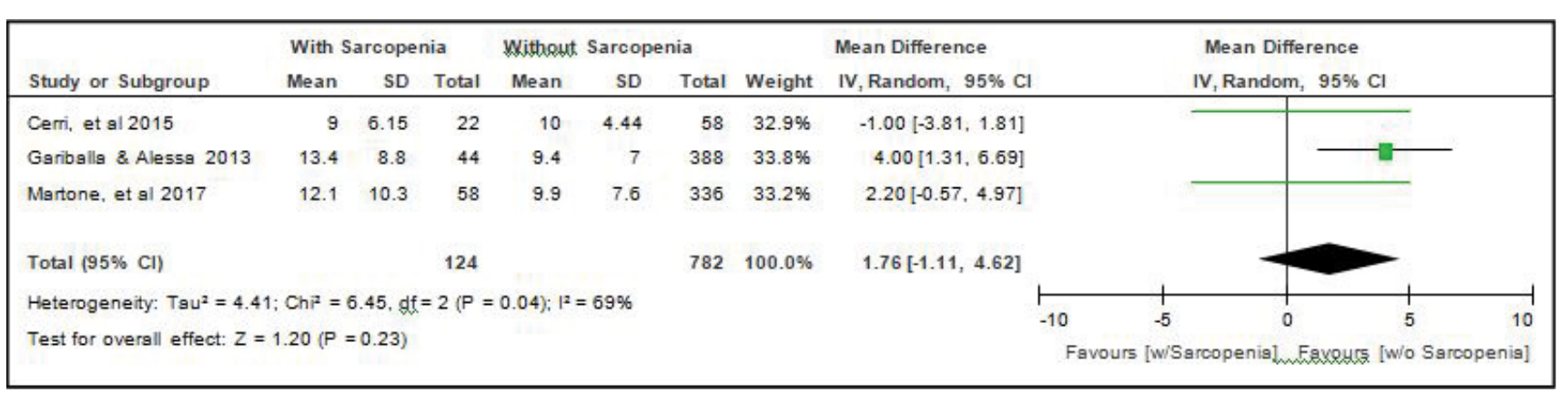

Figure 11: Comparison of length of stay (in days) between patients with or without sarcopenia (random effects model).

smokers among elderly with or without sarcopenia ( $Z$ $=2.77 ; p=0.006$ ) (Figure 9). The study showed that $a$ higher proportion of smokers were noted among those with sarcopenia than those without sarcopenia. The risk of smokers for sarcopenia was $1.26 x$ higher than nonsmokers ( $R R=1.26 ; 95 \% \mathrm{Cl}: 1.07-1.48)$. A fixed effects model was used because there was no significant $(p=$ 0.73 ) heterogeneity noted in the studies included and the $\mathrm{I}_{2}=0 \%$.

Comparison of alcohol drinking between elderly patients with or without sarcopenia: Three studies $[14,17,18]$ gave data on the comparison of alcohol drinking among elderly hospitalized patients with or without sarcopenia, and the analysis of pooled data demonstrated that there was a significant difference noted in the proportion of alcohol drinkers among elderly with or without sarcopenia ( $Z=1.93 ; p=0.05$ ) (Figure 10). The study showed that a lower proportion of alcohol drinkers were noted among those with sarcopenia than those without sarcopenia ( $\mathrm{RR}=0.78 ; 95 \% \mathrm{Cl}$ : 0.61-1.00). A fixed effects model was used because there was no significant $(p=0.93)$ heterogeneity noted in the studies included and the $\mathrm{I}_{2}=0 \%$.

\section{Comparison of outcomes}

Comparison of Length of Stay (in days) between elderly patients with or without sarcopenia: All six studies [4,14-18] compared length of stay between elderly hospitalized patients with or without sarcopenia and the analysis of pooled data demonstrated that there was no significant difference noted using the random effects model ( $Z=1.20 ; p=0.23$ ) (Figure 11). A random effects model was used because there was a significant ( $p=0.04$ ) heterogeneity noted in the studies included and the $I_{2}=69 \%$.

Comparison of mortality between elderly patients with or without sarcopenia: Four studies $[14,15,17,18]$ compared data on mortality among elderly hospitalized patients with or without sarcopenia, and the analysis of pooled data demonstrated that there was a significant difference noted $(Z=6.13 ; p<0.00001)$ (Figure 12). The study showed that risk for mortality among elderly patients with sarcopenia was almost $3 x$ higher than those without sarcopenia (RR $=2.69 ; 95 \% \mathrm{Cl}: 1.96-3.69)$. A fixed effects model was used because there was no significant $(p=0.56)$ heterogeneity noted in the studies included and the $\mathrm{I}_{2}=0 \%$.

Comparison of readmission between elderly patients with or without sarcopenia: Two studies $[14,18]$ compared data on readmission among elderly hospitalized patients with or without sarcopenia, and the analysis of pooled data demonstrated that here was a significant difference noted $(Z=4.94 ; p<0.00001)$ (Figure 13). The study showed that risk for readmission among elderly patients with sarcopenia was $1.48 \mathrm{x}$ higher than those without sarcopenia $(R R=1.48 ; 95 \% \mathrm{Cl}$ : 1.27-1.72). A fixed effects model was used because there was no significant $(p=0.20)$ heterogeneity noted in the studies included although the $I_{2}=38 \%$.

\section{Discussion}

A total of 398 relevant published literatures were 


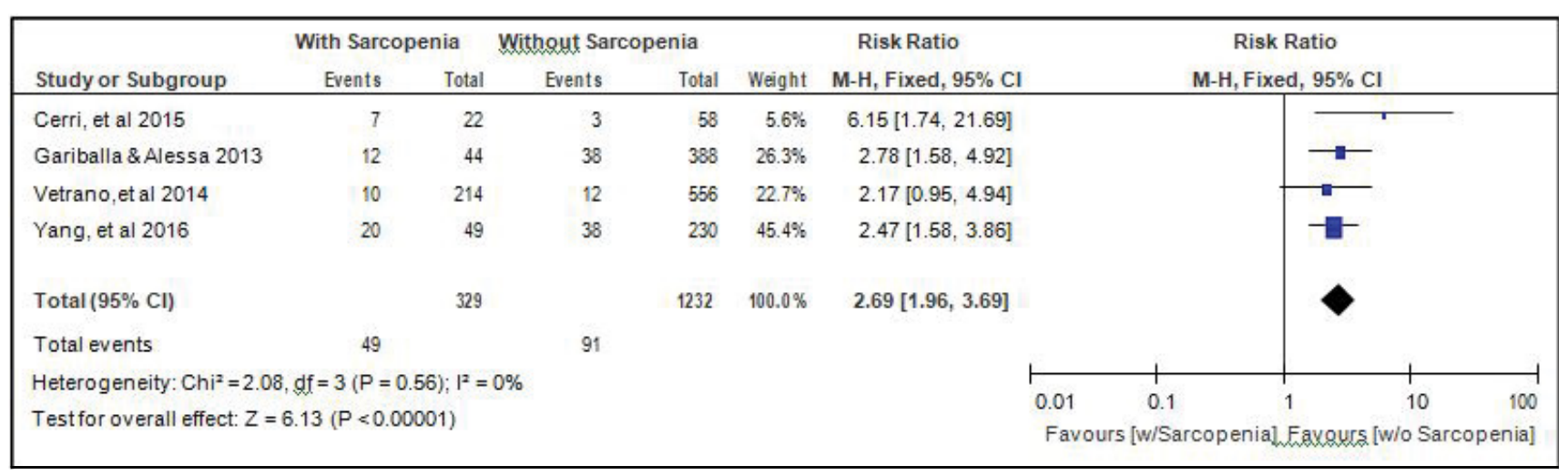

Figure 12: Comparison of mortality between patients with or without sarcopenia (fixed effects model).

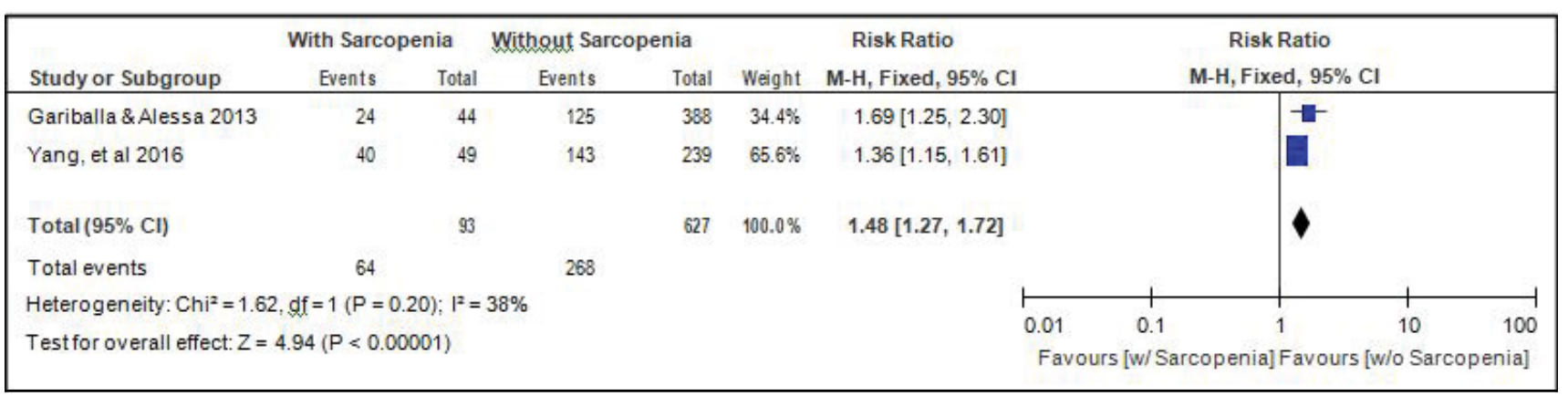

Figure 13: Comparison of readmission between patients with or without sarcopenia (fixed effects model).

searched in PubMed, MEDLINE, and Cochrane. Six observational studies were included in the metaanalysis after screening [4,14-18]. The 6 included studies were of good quality based from the NOS score. Of the 2106 hospitalized elderly patients, 418 (19.8\%) had sarcopenia. The results of this meta-analysis showed that the age of elderly patients with sarcopenia was significantly higher than those without sarcopenia.

In some of the analysis, heterogeneity was noted among the included studies. However, in majority of the analysis, heterogeneity was not observed. This is due to the uniform criteria for the identification of sarcopenia among the elderly patients and the meta-analysis only included a specific group of patients that is elderly with acute sarcopenia. In some studies, the heterogeneity is usually due to the inconsistency in the study design, especially in terms of the selection of participants, interventional strategy, and criteria for identifying outcomes.

Our systematic review has a series of strengths. We conducted the review according to the recommendations stated in The Cochrane Handbook for Systematic Review of Interventions. We set predefined participants, outcomes, and comparisons in order, to avoid biases in the review process. As to the author's belief, we performed an extensive literature search to identify relevant studies based on our predefined inclusion criteria.

However, any meta-analysis is only as good as the constituent studies. All studies included in our analysis may have had methodological limitations. Several operational definitions of sarcopenia are currently proposed in the scientific literature. Although the definition proposed by the EWGSOP is one of the most widely used in current epidemiological studies, it still needs to obtain scientific validation and be recognized as able to predict the health and clinical outcomes of sarcopenia. The present systematic review provides key elements favorable to this validation. Indeed, the majority of studies identified by this systematic review showed an association between sarcopenia, as defined by the EWGSOP, and health-related clinical outcomes such as mortality and readmission.

Although the included studies were not significantly heterogeneous, given the small number of studies, statistical conclusions on determinants of heterogeneity might be flawed. Further, we cannot fully exclude publication bias. Although a statistical test for the detection of publication bias can be performed, however, because there are $<10$ studies, we are aware that these tests have very low power in the meta-analysis of only a few studies. To limit the risk of publication bias, we did not impose restrictions by language on the publication.

\section{Conclusion}

In summary, this meta-analysis showed that the prevalence of acute sarcopenia among elderly hospitalized patients was $19.8 \%$. The age of elderly patients with sarcopenia was significantly higher than 
those without sarcopenia while the BMI was lower. The proportion of smokers was also higher among elderly patients with sarcopenia. Moreover, the results of this meta-analysis showed that the length of stay was not significantly different between elderly patients with or without sarcopenia. However, mortality and readmission rates were significantly higher among elderly patients with acute sarcopenia.

\section{References}

1. Beaudart $C$, Zaaria $M$, Pasleau $F$, Reginster JY, Bruyère O (2017) Health Outcomes of Sarcopenia: A Systematic Review and Meta-Analysis. PLoS One 12: e0169548.

2. Delmonico MJ, Harris TB, Lee JS, Visser M, Nevitt M, et al. (2007) Alternative definitions of sarcopenia, lower extremity performance, and functional impairment with aging in older men and women. J Am Geriatr Soc. 55: 769-774.

3. Goodpaster BH, Park SW, Harris TB, Kritchevsky SB, Nevitt M, et al. (2006) The loss of skeletal muscle strength, mass, and quality in older adults: The health, aging and body composition study. J Gerontol A Biol Sci Med Sci 61: 1059-1064.

4. Martone AM, Bianchi L, Abete P, Bellelli G, Bo M, et al (2017) The incidence of sarcopenia among hospitalized older patients: Results from the Glisten study. J Cachexia Sarcopenia Muscle 8: 907-914.

5. Han A, Bokshan S, Marcaccio S, DePasse J, Daniels A (2018) Diagnostic Criteria and Clinical Outcomes in Sarcopenia Research: A Literature Review. J Clin Med 7: 70.

6. Walston J (2012) Sarcopenia in older adults. Curr Opin Rheumatol 24: 623-627.

7. Welch C, Smith ZKH, Greig CA, Lord JM, Jackson TA (2018) Acute Sarcopenia Secondary to HospitalisationAn Emerging Condition Affecting Older Adults. Aging \& Disease 9: 151-164.

8. Kortebein P, Ferrando A, Lombeida J, Wolfe R, Evans WJ (2007) Effect of 10 days of bed rest on skeletal muscle in healthy older adults. JAMA 297: 1772-1774.
9. Jonesn PD, Moore SM, Urban RJ, Sanford AP, Aarsland A, et al (2004) Essential amino acid and carbohydrate supplementation ameliorates muscle protein loss in humans during 28 days bedrest. J Clin Endocrinol Metab 89: 4351-4358.

10. Shafiee G, Keshtkar A, Soltani A, Ahadi Z, Larijani B, et al. (2017) Prevalence of sarcopenia in the world: A systematic review and meta- analysis of general population studies. $J$ Diabetes Metab Disord 16: 21.

11. Beaudart C, Rizzoli R, Bruyere O, Reginster J, Biver E (2014) Sarcopenia: Burden and challenges for public health. Arch Public Health 72: 45.

12. Chen LK, Liu LK, Woo J, Assantachai P, Auyeung TW, et al. (2014) Sarcopenia in Asia: Consensus report of the asian working group for sarcopenia. J Am Med Dir Assoc 15: $95-101$.

13. Alfonso AJC, Baeyens JP, Bauer JM, Boirie Y, Cederholm, et al. (2010) Sarcopenia: European consensus on definition and diagnosis: Report of the European Working Group on Sarcopenia in Older People. Age and ageing 39: 412-423.

14. Gariballa S, Alessa A (2013) Sarcopenia: Prevalence and prognostic significance in hospitalized patients. Clin Nutr 32: $772-776$

15. Cerri AP, Bellelli G, Mazzone A, Pittella F, Landi F, et al. (2015) Sarcopenia and malnutrition in acutely ill hospitalized elderly: Prevalence and outcomes. Clin Nutr 34: 745-751.

16. Rossi AP, Fantin F, Micciolo R, Bertocchi M, Bertassello $P$, et al. (2014) Identifying sarcopenia in acute care setting patients. J Am Med Dir Assoc 15: e7-e12.

17. Vetrano DL, Landi F, Volpato S, Corsonello A, Meloni E, et al. (2014) Association of sarcopenia with short- and longterm mortality in older adults admitted to acute care wards: Results from the CRIME study. J Gerontol A Biol Sci Med Sci 69: 1154-1161.

18. Yang M, Hu X, Wang H, Zhang L, Hao Q, et al. (2017) Sarcopenia predicts readmission and mortality in elderly patients in acute care wards: A prospective study. J Cachexia Sarcopenia Muscle 8: 251-258. 